\title{
Flüssigkeitsanalyse mittels mikrothermischer Sensoren
}

\author{
Bastian Schmitt, Christian Kiefer, Karsten Kühn und Andreas Schütze \\ Universität des Saarlandes, Lehrstuhl für Messtechnik (LMT), Saarbrücken, Campus A5.1
}

\section{Zusammenfassung}

Die Analyse von Flüssigkeitsgemischen bezüglich ihrer Zusammensetzung über einen großen Konzentrationsbereich stellt noch immer eine Herausforderung dar. Das Spektrum reicht von der Erfassung von Methanol in Wasser für Direktmethanolbrennstoffzellen über die Ölqualitätsmessung bis hin zur Erfassung von Verunreinigungen in Lebensmitteln. Hierfür wird ein Sensorprinzip untersucht, das unterschiedliche thermische Eigenschaften wie die resultierende Wärmeleitfähigkeit und -kapazität in der Mischung erfasst. Vor diesem Hintergrund wurde ein Analysesystem, bestehend aus Messkammer, Spritzenpumpen zur Fluiddosierung als auch entsprechenden Sensoren konzipiert und realisiert. In ersten Versuchen bestätigte sich die Anwendbarkeit des Systems.

\section{Konzept}

In ersten Messungen werden zwei Flüssigkeiten mit unterschiedlichen thermischen Eigenschaften vor der in Bild 1 gezeigten Messkammer aus PVDF gemischt und anschließend in die Kammer geleitet, in der das Mischungsverhältnis durch Messung der thermischen Eigenschaften bestimmt wird. Dies geschieht durch einen Temperaturpuls, eingeprägt durch einen zentral auf der Sensorplatine angeordneten Heizer, dessen Ausbreitung im Medium über vier um den Heizer angeordnete Widerstandsstrukturen gemessen wird. Neben der statischen Messung der Temperaturverteilung soll auch das Zeitverhalten des Temperaturpulses ausgewertet werden, um dadurch Informationen sowohl über die Wärmeleitfähigkeit als auch Wärmekapazität der Mischung zu gewinnen. Derzeit werden zwei Aufbauvarianten verfolgt, einerseits eine über SMD-Widerstände realisierte Sensorplatine (Makromodell), andererseits ein mittels Fotolithografie strukturierter Sensorchip (Mikromodell). Beide Varianten sind in Bild 3 schematisch dargestellt.

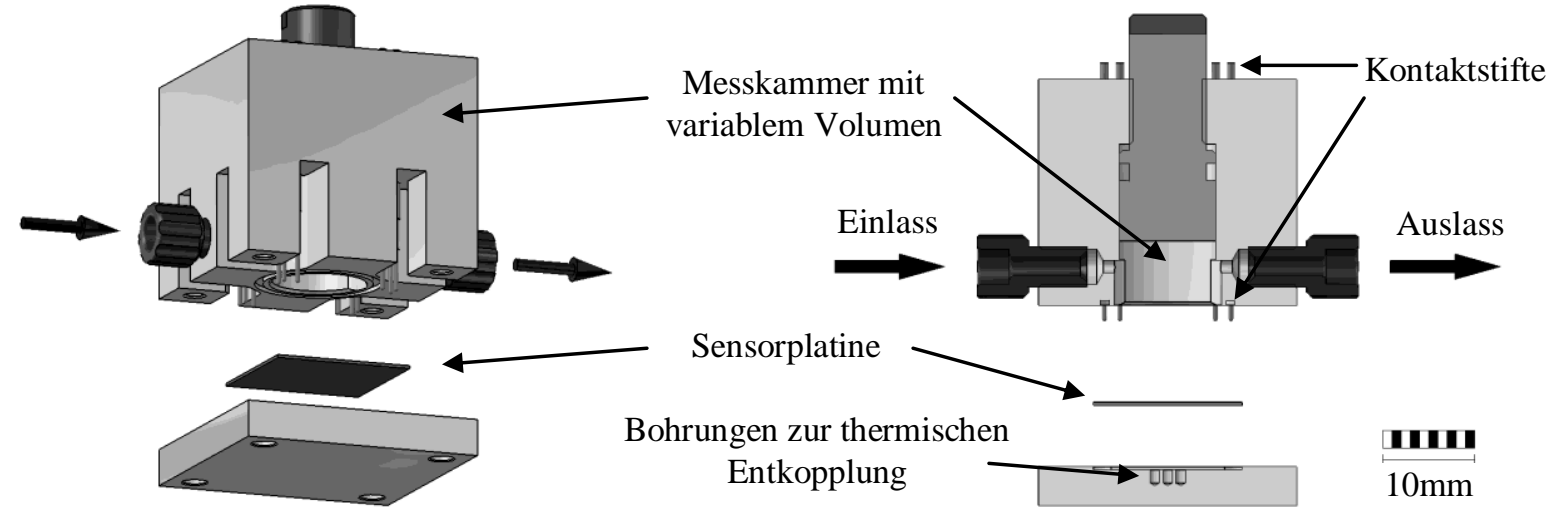

Bild 1 Messkammer mit Sensorplatine. Das Volumen kann variabel durch einen in der Mitte befindlichen Stößel angepasst werden. Zur elektrischen Kontaktierung werden Kontaktstifte genutzt, die durch die Messkammer hindurch zur Sensorplatine geführt sind

\section{Messaufbau}

Der gesamte Messaufbau ist in Bild 2 dargestellt. Zur Erzeugung der zu messenden Flüssigkeitsgemische sind Spritzenpumpen vorhanden, mit denen sich Verhältnisse von bis zu 1:500.000 erzeugen lassen. Die Gemische werden in die dargestellte Mischkammer geleitet und bedecken den in Bild 3 dargestellten Sensor. 
Durch den Heizer in der Mitte der Sensoren wird ein während der Heizphase konstanter Strom geleitet und somit ein Temperaturpuls eingeprägt. Mittels Vierleitermessung kann durch den Spannungsabfall am Heizer dessen Widerstand und damit dessen Temperatur exakt bestimmt werden. Zusätzlich wird mittels der vier Temperatursensoren die Temperaturverteilung gemessen. Die Signalauswertung erfolgt in LabVIEW, wobei die Messdaten mit einem Datenerfassungsmodul (NI USB-6008, Fa. National Instruments) erfasst werden.

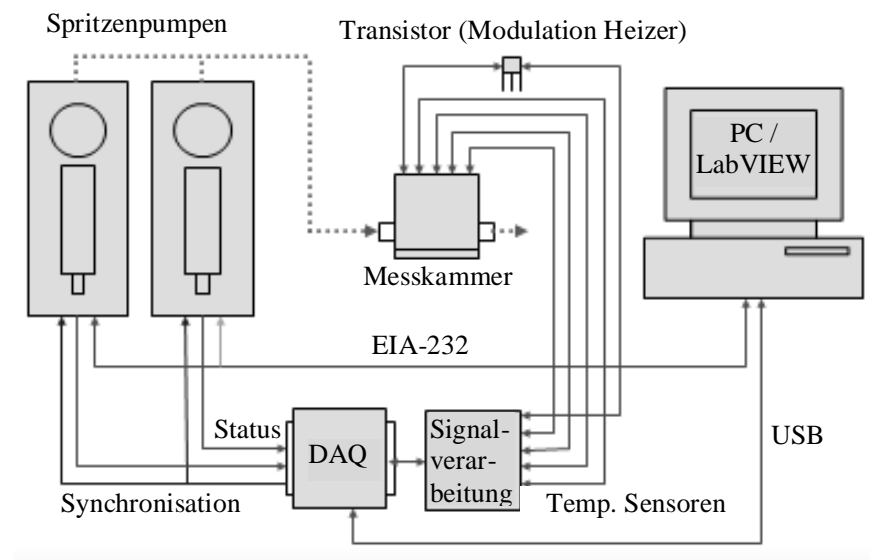

Bild 2 Messaufbau mit Signalerfassung bestehend aus Spritzenpumpen zur Erzeugung eines definierten Gemischs, Messkammer, PC mit LabVIEW zur Ansteuerung und Messdatenauswertung sowie DAQ und Signalverarbeitungselektronik zur Signalerfassung

\subsection{Aufbau der Sensoren}

Die mikrostrukturierten Sensoren aus Bild 3 links bestehen aus auf Borosilikatglas gesputtertem Titan. Die Schichtdicke der resistiven Schicht beträgt etwa 0,5 $\mu \mathrm{m}$. Die Abmessungen der temperaturempfindlichen Widerstandselemente wurden so ausgelegt, dass zur Verringerung der Eigenerwärmung und somit zur Vermeidung einer Verfälschung des Ergebnisses Grundwiderstände von etwa $2 \mathrm{k} \Omega$ erreicht wurden. Der Widerstand des kreisförmigen Heizers in der Mitte der Chips kann während des Heizens mittels Vierleitermessung exakt bestimmt werden, um dadurch die genaue Temperatur bzw. Leistung regeln zu können. Zum Testen eines low-cost-Ansatzes wurde zusätzlich eine mittels SMD-Widerständen realisierte Sensorplatine wie in Bild 3 rechts $\mathrm{zu}$ sehen erstellt. Es wurden verschiedene Sensorgeometrien mit variierenden Abmessungen der Heizer und Abständen $\mathrm{zu}$ den temperaturempfindlichen Widerständen realisiert, um den Einfluss dieser Größen testen zu können.
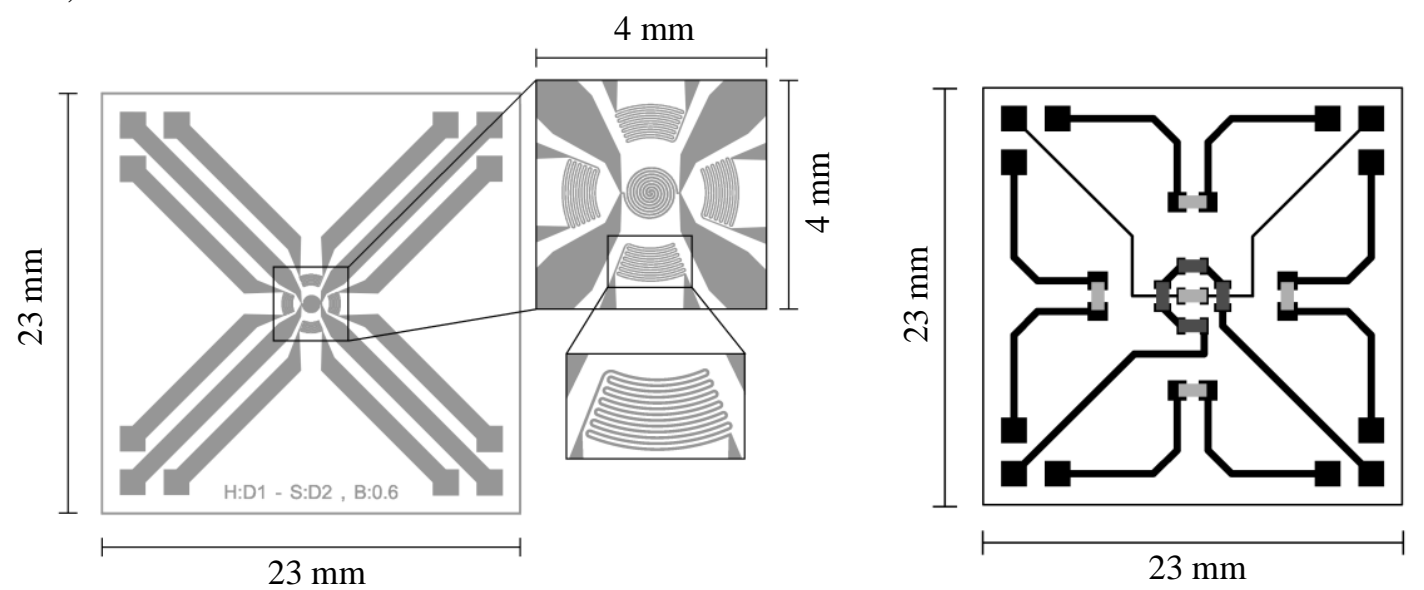

Bild 3 Links: Draufsicht des mikrostrukturierten Sensoraufbaus mit Detailansicht des Heizers sowie der vier temperatur-empfindlichen Widerstandselemente

Rechts: Draufsicht eines SMD-Chipaufbaus. In der Mitte dargestellt ist ein Ring aus SMD-Schichtwiderständen zum Einprägen eines Temperaturpulses mit einem zentralen SMD-Temperaturwiderstand. Außen dargestellt sind Temperaturwiderstände zur lokalen Messung der Temperaturverteilung 
So beträgt der Heizerdurchmesser zwischen 0,5 und $1 \mathrm{~mm}$, die Abstände zwischen Heizer und Sensor betragen zwischen 0,5 und $2 \mathrm{~mm}$. Wie die ersten Ergebnisse zeigen, siehe Abschnitt 4, hat dieser Abstand einen großen Einfluss auf die Selektivität der Messung. Zusätzlich wurden auch Chips realisiert, auf denen die einzelnen Temperatursensoren unterschiedliche Abstände zum Heizer aufweisen, um - bei Annahme einer symmetrischen Temperaturverteilung - zusätzliche Informationen gewinnen zu können. Auf der Sensorplatine mit SMD-Bauteilen wird die Temperatur durch den inneren Ring von Widerständen eingeprägt. Der Widerstand im Zentrum des Chips dient der Temperaturmessung der vier Heizerwiderstände. Die Anordnung der Kontaktpads auf den Sensoren entspricht der der Durchkontaktierungen der Messkammer.

\subsection{Bestimmung des Temperaturkoeffizienten von Titan}

Um die Temperatur mit den mikrostrukturierten Sensoren exakt messen zu können musste in einem ersten Schritt der Temperaturkoeffizient des elektrischen Widerstands der Titan-Dünnschicht bestimmt werden. Hierfür wurden die mikrostrukturierten Chips in einem Klimaschrank in $10{ }^{\circ} \mathrm{C}$-Schritten von $20^{\circ} \mathrm{C}$ bis $80^{\circ} \mathrm{C}$ erwärmt und dabei der elektrische Widerstand der je vier einzelnen Widerstandsstrukturen sowie des Heizers erfasst, einen Auszug der dabei ermittelten Werte für sechs Sensorstrukturen auf unterschiedlichen Chips zeigt Bild 4. Der sich daraus ergebende lineare Temperaturkoeffizient $\alpha_{0}$ bei einer Referenztemperatur $\mathrm{T}_{0}=0{ }^{\circ} \mathrm{C}$ ergibt sich somit gemäß $\mathrm{R}(\mathrm{T})=\mathrm{R}\left(\mathrm{T}_{0}\right) \cdot\left(1+\alpha_{0}\left(\mathrm{~T}-\mathrm{T}_{0}\right)\right)$ durch Linearisierung der Kennlinien, siehe Bild 4 rechts. Somit kann die Temperatur bis auf weniger als $\pm 2{ }^{\circ} \mathrm{C}$ genau gemessen werden.
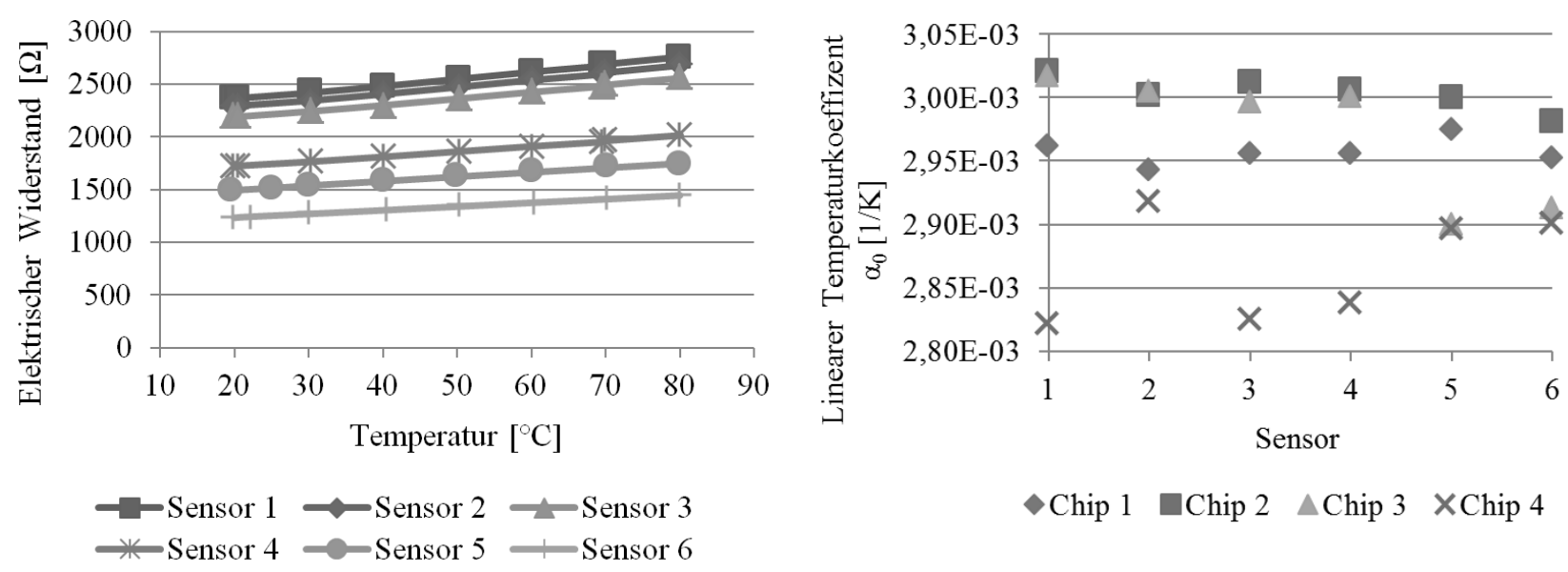

$\triangle$ Chip $1 \quad$ Chip $2 \triangle$ Chip $3 \quad$ XChip 4

Bild 4 Abhängigkeit des elektrischen Widerstands der Titanstrukturen von der Temperatur und resultierender linearer Temperaturkoeffizient $\alpha_{0}$

\section{Simulationsergebnisse}

Zur Abschätzung der erzielbaren Messwerte wurde das Problem in COMSOL Multiphysics (Fa. Comsol AB) simuliert. Hierbei zeigte sich entgegen den Erwartungen, dass bei einer Zunahme der Wärmeleitfähigkeit des Flüssigkeitsgemischs die an den vier Sensoren gemessene Temperatur abnimmt. Dies resultiert daraus, dass das verwendete Borosilikatglas mit $1,2 \mathrm{~W} / \mathrm{m} / \mathrm{K}$ eine etwa doppelt so große Wärmeleitfähigkeit wie das zu messende Gemisch aufweist. Somit findet die Wärmeleitung hauptsächlich im Substrat statt. Steigt nun die Wärmeleitfähigkeit des Gemischs, so wird mehr Energie durch das Medium abgeführt und die Temperaturen der Sensoren steigen weniger an. Des Weiteren zeigte sich ein starker Einfluss von Konvektion innerhalb der Mischkammer bei Kammerhöhen über $1 \mathrm{~mm}$. Diese bewirkt, dass das erwärmte Gemisch über dem Heizer aufsteigt und von den Seiten der Kammer die nicht erwärmte Flüssigkeit nachströmt. Somit werden die Widerstände zunächst von der kalten Flüssigkeit überströmt, was sich negativ auf die Messung auswirkt. Die Simulation zeigt damit, dass die Sensoranordnung idealerweise auf einem Membransubstrat realisiert werden sollte, um die störende Wärmeleitung durch das Substrat zu minimieren, alternativ könnte evtl. auch Kunststoff genutzt werden. Zudem sollten kleine Kammerhöhen verwendet werden um die Konvektion zu unterbinden. 


\section{Messergebnisse}

Zur Ansteuerung des Heizers wurde für erste Versuche ein rechteckiger Signalverlauf mit einer Amplitude von $2 \mathrm{~mA}$ bei einem Offset von ebenfalls $2 \mathrm{~mA}$ gewählt. Die Frequenz betrug $0,25 \mathrm{~Hz}$ bei einem Tastverhältnis von $25 \%$. Je nach Heizergrundwiderstand ergibt sich somit eine eingeprägte Heizleistung von etwa $32 \mathrm{~mW}$, was trotz des thermischen Messprinzips einen sehr geringen Wert darstellt. Eine einzelne Messperiode ist in Bild 5 links dargestellt. Bei der Zugabe von Methanol zu Wasser zeigt sich eine Zunahme der gemessenen Temperatur. Dieser Trend deckt sich mit den Simulationsergebnissen, da bei Zunahme des Methanolanteils die Wärmeleitfähigkeit des Gemisches sinkt. In Bild 5 rechts ist der Einfluss des Abstandes der Sensoren zum Heizer zu erkennen. Dabei wurde die Spannungsdifferenz vor und am Ende der Heizphase bestimmt und für unterschiedliche Mischungsverhältnisse in Abhängigkeit des Abstands vom Heizer zum Sensor aufgetragen. Es ist zu erkennen, dass die Selektivität bei kleineren Abständen steigt. Weitere Versuche sollen auch das Zeitverhalten betrachten, um neben der Wärmeleitfähigkeit auch die Wärmekapazität als Messgröße auszunutzen, um damit eine höhere Selektivität zu erzielen. Jeweils zu Beginn und Ende des Heizvorgangs sind starke Überschwinger erkennbar, die mit steigendem Methanolanteil abnehmen und im Zuge der weiterführenden Arbeit ebenfalls zur Auswertung herangezogen werden sollen. Die Ursache dieser Überschwinger wird durch weitere Messungen untersucht.
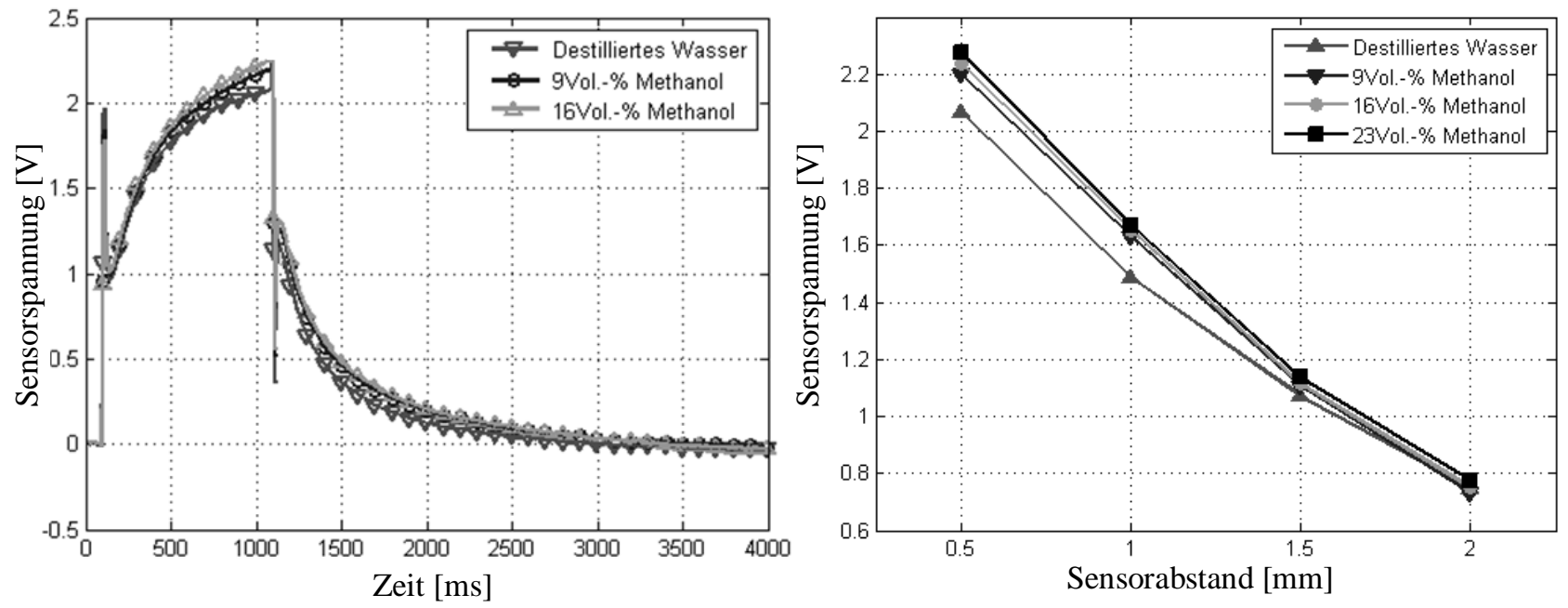

Bild 5 Links: Darstellung einer Messperiode bei unterschiedlichen Mischungsverhältnissen von Methanol und Wasser bei einem Sensorabstand von $0,5 \mathrm{~mm}$. Rechts: Vergleich des Sensorsignals bei unterschiedlichen Abständen der Sensoren zum Heizer

\section{Ausblick}

Die Ergebnisse der ersten Messungen bezüglich der Geometrie der Sensorstrukturen werden genutzt um neue Sensoren mit größerer Empfindlichkeit und Selektivität herzustellen. Die Ergebnisse der Simulation und der ersten Messungen bestätigen den Ansatz und zeigen insbesondere das Optimierungspotential durch Miniaturisierung der Sensoren auf. Nach der Bestimmung der Ursache der Überschwinger jeweils zu Beginn und Ende der Heizphase wird überprüft, ob die Auswertung dieser mit in den Algorithmus zur Bestimmung des Mischungsverhältnisses aufgenommen werden kann.

Das gewählte Spritzenpumpensystem ermöglicht zudem die Realisierung von Mischungsverhältnissen bis zu 1:500.000. Um auch sehr kleine Beimengungen bzw. Verunreinigungen erfassen zu können, soll das mikrothermische Sensorsystem ergänzt werden durch Messung der dielektrischen Eigenschaften. Hierbei sollen neuartige nanostrukturierte Sensoren zum Einsatz kommen, die ein Fractional Order Verhalten zeigen [1].

\section{Literatur}

[1] K. Biswas, S. Sen, P.K. Dutta: Realization of a Constant Phase Element and Ist Performance Study in a Differentiator Circuit, IEEE Trans. Circuits and Systems II: express briefs, Band 53, Nr. 9, 2006. 